\title{
Looking at Quantization of a Wave Function, from Weber (1961), to Signals from Wavefunctions at the Mouth of a Wormhole
}

\author{
Andrew Beckwith \\ Physics Department, College of Physics, Chongqing University, Chongqing, China \\ Email: rwill9955b@gmai.com
}

How to cite this paper: Beckwith, A. (2021) Looking at Quantization of a Wave Function, from Weber (1961), to Signals from Wavefunctions at the Mouth of a Wormhole. Journal of High Energy Physics, Gravitation and Cosmology, 7, 1037-1048. https://doi.org/10.4236/jhepgc.2021.73062

Received: June 4, 2021

Accepted: July 4, 2021

Published: July 7, 2021

Copyright (c) 2021 by author(s) and Scientific Research Publishing Inc. This work is licensed under the Creative Commons Attribution International License (CC BY 4.0). http://creativecommons.org/licenses/by/4.0/

\begin{abstract}
We utilize how Weber in 1961 initiated the process of quantization of early universe fields to the problem of what may be emitted at the mouth of a wormhole. While the wormhole models are well developed, there is as of yet no consensus as to how, say GW or other signals from a wormhole mouth could be quantized or made to be in adherence to a procedure Weber cribbed from Feynman, in 1961. In addition, we utilize an approximation for the Hubble parameter parameterized from Temperature using Sarkar's $\mathrm{H} \sim \mathrm{Tem}-$ perature relations, as given in the text. Finally, after doing this, we go to the Energy as E also Temperature, and from there use E (energy) as signal frequency. This gives us an idea of how to estimate frequency generated at the mouth of a wormhole.
\end{abstract}

\section{Keywords}

Minimum Scale Factor, Cosmological Constant, Space-Time Bubble, Bouncing Cosmologies

\section{Introduction}

We bring up this study first a result given by Weber, in 1961 [1] as to getting an initial wavefunction given in [2], which may be able to model behavior of what happens in the mouth of a wormhole if we make the assumption given in [3] that $\mathrm{H}$ (Hubbles parameter) is proportional to Temperature, and then go to Energy $\sim$ Temperature. The last part will be enough to isolate, up to first principles a net frequency value.

The behavior of frequency, versus certain conditions at the mouth of a wormhole may give us clues to be investigated later as to polarization states relevant to 
the wormhole [4] as well as examining what may be relevant to measurement of signals from a wormhole [5].

In doing all of this, the idea is that we are evolving from the Einstein-Rosen bridge to a more complete picture of GR which may entail a new representation of the Visser "Chronology protection" paper as in [6] [7].

\section{Looking at the Weber Book as to Reformulate Quantization Imposed in GR}

Using [1] a statement as to quantization for a would be GR term comes straight from:

$$
\Psi_{\text {Later }}=\int \sum_{H} \mathrm{e}^{\left(i I_{H} / \hbar\right)\left(t, t^{0}\right)} \Psi_{\text {Earlier }}\left(t^{0}\right) \mathrm{d} t^{0}
$$

The approximation we are making is to pick one index, so as to have:

$$
\Psi_{\text {Later }}=\int \sum_{H} \mathrm{e}^{\left(i I_{H} / \hbar\right)\left(t, t^{0}\right)} \Psi_{\text {Earlier }}\left(t^{0}\right) \mathrm{d} t^{0} \underset{H \rightarrow 1}{\longrightarrow} \int \mathrm{e}^{\left(i I_{\mathrm{FIXED}} / \hbar\right)\left(t, t^{0}\right)} \Psi_{\text {Earlier }}\left(t^{0}\right) \mathrm{d} t^{0}
$$

This corresponds to say being primarily concerned as to GW generation, which is what we will be examining in our ideas, via using:

$$
\mathrm{e}^{\left(i I_{\mathrm{FIXED}} / \hbar\right)\left(t, t^{0}\right)}=\exp \left[\frac{i}{\hbar} \cdot \frac{c^{4}}{16 \pi G} \cdot \int_{\mathrm{M}} \mathrm{d} t \cdot \mathrm{d}^{3} r \sqrt{-g} \cdot(\Re-2 \Lambda)\right]
$$

We will use the following, namely, if $\Lambda$ is a constant, do the following for the Ricci scalar [8] [9],

$$
\Re=\frac{2}{r^{2}}
$$

If so then we can write the following, namely: Equation (3) becomes, if we have an invariant Cosmological constant, so we write $\Lambda \underset{\text { all time }}{\longrightarrow} \Lambda_{0}$ everywhere, then,

$$
\mathrm{e}^{\left(i I_{H_{\mathrm{FIXED}}} / \hbar\right)\left(t, t^{0}\right)}=\exp \left[\frac{i}{\hbar} \cdot \frac{c^{4} \cdot \pi \cdot t^{0}}{16 G} \cdot\left(r-r^{3} \Lambda_{0}\right)\right]
$$

Then, we have that Equation (1) is rewritten to be:

$$
\begin{aligned}
& \Psi_{\text {Later }}=\int \sum_{H} \mathrm{e}^{\left(i I_{H} / \hbar\right)\left(t, t^{0}\right)} \Psi_{\text {Earlier }}\left(t^{0}\right) \mathrm{d} t^{0} \\
& \underset{\text { at wormhole }}{\longrightarrow} \int \exp \left[\frac{i}{\hbar} \cdot \frac{c^{4} \cdot \pi \cdot t^{0}}{16 G} \cdot\left(r-r^{3} \Lambda_{0}\right)\right] \Psi_{\text {Earlier }}\left(t^{0}\right) \mathrm{d} t^{0}
\end{aligned}
$$

\section{Examining the Behavior of the Earlier Wavefunction in Equation (6)}

[2] states a Hartle-Hawking wavefunction which we will adapt for the earlier wavefunction as stated in Equation (6) so as to read as follows:

$$
\Psi_{\text {Earlier }}\left(t^{0}\right) \approx \Psi_{H H} \propto \exp \left(\frac{-\pi}{2 G H^{2}} \cdot(1-\sinh (H t))^{3 / 2}\right)
$$


Here, making use of Sarkar [3], we set, if say $g_{*}$ is the degree of freedom allowed [9],

$$
H=1.66 \sqrt{g_{*}} T_{\text {temp }}^{2} / M_{\text {Planck }}
$$

We assume initially a relatively uniformly given temperature, that $H$ is constant.

So then we will be attempting to write out an expansion as to what Equation (6) gives us while we use Equation (7) and Equation (8), with $\mathrm{H}$ approximately constant.

\section{Methods Used in Calculating Equation (6), with Interpretation of the Results}

We will be considering how to express Equation (6) and in doing this we will be looking at having a constant value for Equation (8). If so then,

$$
\Psi_{\text {Later }}=\int \exp \left[\frac{i}{\hbar} \cdot \frac{c^{4} \cdot \pi \cdot t^{0}}{16 G} \cdot\left(r-r^{3} \Lambda_{0}\right)\right] \exp \left(\frac{-\pi}{2 G H^{2}} \cdot(1-\sinh (H t))^{3 / 2}\right) \mathrm{d} t^{0}
$$

Then using numerical integration, [10] [11] [12] on page 751 of this [12] citation:

$$
\begin{aligned}
& \Psi_{\text {Later }} \frac{t_{M} \rightarrow \varepsilon^{+}}{t_{M}} \int_{0}^{i \cdot(\tilde{\alpha} 1) \cdot t(\tilde{\alpha} 2) \cdot(1-\sinh (H t))^{3 / 2}} \mathrm{~d} t \\
& \approx \frac{t_{M}}{2} \cdot\left(\mathrm{e}^{i \cdot(\tilde{\alpha} 1) \cdot t_{M}-(\tilde{\alpha} 2) \cdot\left(1-\sinh \left(H \cdot t_{M}\right)\right)^{3 / 2}}-1\right) \\
& \tilde{\alpha} 1=\left[\frac{c^{4} \cdot \pi}{16 G \hbar} \cdot\left(r-r^{3} \Lambda_{0}\right)\right], \quad \tilde{\alpha} 2=\frac{\pi}{2 G H^{2}}
\end{aligned}
$$

Notice the terms for the $H$ factor, and from here we will be making our prediction.

If the energy, $E$, has the following breakdown,

$$
\begin{aligned}
& H=1.66 \sqrt{g_{*}} T_{\text {temp }}^{2} / M_{\text {Planck }} \\
& \Rightarrow E \approx k_{B} T_{\text {Temp }} \approx \hbar \cdot \omega_{\text {signal }} \\
& \Rightarrow \omega_{\text {signal }} \approx \frac{k_{B} \cdot \sqrt{M_{\text {Planck }} H}}{\hbar \cdot \sqrt{1.66 \sqrt{g_{*}}}}
\end{aligned}
$$

The upshot is that we have, in this, a way to obtain a signal frequency by looking at the real part of Equation (11) above, if we have a small $t$, initially (small time step).

\section{How to Compare with a Kieffer Solution and Thereby Isolate the Cosmological Constant Contribution}

Equation (11) would imply an initial frequency dependence. What we are doing next is to strategize as to understand the contribution of the cosmological constant in this sort of problem. I.e., the way to do it would be to analyze a Kieffer "dust solution" as a signal from the Wormhole. I.e., look at [13], where we as- 
sume that $t$, would be in this case the same as in Equation (10) above. I.e. in this case we will write having

$$
\Delta \omega_{\text {signal }} \Delta t \approx 1
$$

If so then we can assume, that the time would be small enough so that:

$$
\Delta t \approx \frac{\hbar \sqrt{1.66 \sqrt{g_{*}}}}{k_{B} \cdot \sqrt{M_{\text {Planck }} H}}
$$

If Equation (13) is of a value somewhat close to $t$, in terms of general initial time, we can write

$$
\psi_{\tilde{n}, \lambda}(t, r) \equiv \frac{1}{\sqrt{2 \pi}} \cdot \frac{\tilde{n} ! \cdot(2 \lambda)^{\tilde{n}+1 / 2}}{\sqrt{(2 \tilde{n}) !}} \cdot\left[\frac{1}{(\lambda+i \cdot t+i \cdot r)^{\tilde{n}+1}}-\frac{1}{(\lambda+i \cdot t-i \cdot r)^{\tilde{n}+1}}\right]
$$

Here the time $t$ would be proportional to Planck time, and $r$ would be proportional to Planck length, whereas we set

$$
\lambda \approx \sqrt{\frac{8 \pi G}{V_{\text {volume }} \hbar^{2} t^{2}}} \underset{G=\hbar=\ell_{\text {Planck }}=k_{B}=1}{\longrightarrow} \sqrt{\frac{8 \pi}{t^{2}}} \equiv \frac{\sqrt{8 \pi}}{t}
$$

Then a preliminary emergent space-time wavefunction would take the form of:

$$
\begin{aligned}
& \psi_{\tilde{n}, \lambda}(\Delta t, r) \equiv \frac{1}{\sqrt{2 \pi}} \cdot \frac{\tilde{n} ! \cdot\left(2 \cdot \sqrt{8 \pi} \cdot(\Delta t)^{-1}\right)^{\tilde{n}+1 / 2}}{\sqrt{(2 \tilde{n}) !}} \\
& \cdot\left[\frac{1}{\left(\sqrt{8 \pi} \cdot(\Delta t)^{-1}+i \cdot \Delta t+i \cdot r\right)^{\tilde{n}+1}}-\frac{1}{\left(\sqrt{8 \pi} \cdot(\Delta t)^{-1}+i \cdot \Delta t-i \cdot r\right)^{\tilde{n}+1}}\right]
\end{aligned}
$$

Just at the surface of the bubble of space-time, with $t_{\text {Planck }} \propto \Delta t$, and $r \propto \ell_{\text {Planck }}$.

This is from a section, page 239 of the $3^{\text {rd }}$ edition of Kieffer's book, as to a quantum theory of collapsing dust shells. And so then we have the following procedure as to isolate out the contribution of the Cosmological constant. Namely, take the real part of Equation (16) and compare it with the Real part of Equation (10).

Another way to visualize this situation, and this is a different way to interpret Equation (15). To do so we examine looking at page 239 of Kieffer, namely [13] where one has an expectation value to energy we can write as

$$
\langle E\rangle_{\kappa=n, \lambda}=\frac{(\kappa=n)+1 / 2}{\lambda} \underset{\lambda \approx 1 / \hbar \omega}{\longrightarrow} \hbar \omega \cdot((\kappa=n)+1 / 2)
$$

What we can do, is to ascertain the last step would be to make Equation (16) in a sense partly related to the simple harmonic oscillator. But we should take into consideration the normalization using that if $\hbar=\ell_{P}=G=t_{P}=k_{B}=1$ is done via Plank unit normalization [14] [15]. If so, then we have that frequency is proportional to $1 / t$, where $t$ is time. I.e. hence if there is a value of $n=0$ and 
making use of the frequency, we then would be able to write Equation (16) as

$$
\Psi_{1, \kappa=n=0} \approx \sqrt{\frac{\omega}{\pi}} \cdot\left[\frac{1}{\omega+i \cdot(t+r)}-\frac{1}{\omega+i \cdot(t-r)}\right]
$$

Or,

$$
\Psi_{2, \kappa=n=0} \approx \frac{1}{\sqrt{\pi}} \sqrt{\frac{\sqrt{8 \pi}}{t}} \cdot\left[\frac{1}{\frac{\sqrt{8 \pi}}{t}+i \cdot(t+r)}-\frac{1}{\frac{\sqrt{8 \pi}}{t}+i \cdot(t-r)}\right]
$$

With, say

$$
\omega \approx \frac{\sqrt{8 \pi}}{t}
$$

And this in a setting where we have the dimensional reset of Planck Units [14] [15],

$$
\hbar=\ell_{P}=G=t_{P}=k_{B}=1
$$

\section{Formal Bounding of the Cosmological Constant, in Terms of Two Wavefunctions Plus Analysis of Initial Wormhole Frequency Values}

To do this we, first of all, consider the real part of Equation (18) when $n$ is set equal to zero, and the state should be the same as the real part of Equation (10). Then do the same with the real part of Equation (19) and compare it with Equation (10) in order to isolate out a bound to the cosmological constant.

Doing so, leads to the following situation, and keep in mind that we are using Equation (21) for regularization as well as setting

$$
r \equiv \widehat{B} \cdot r_{P} \underset{r_{P} \rightarrow 1}{\longrightarrow} \widehat{B}
$$

If so, then we have the following bounding as far as the value of the cosmological "constant", namely,

$$
\begin{aligned}
& \Psi_{\text {Later }} \underset{t_{M} \rightarrow \varepsilon^{+}}{\longrightarrow} \int_{0}^{t_{M}} \mathrm{e}^{i \cdot(\tilde{\alpha} 1) \cdot t(\tilde{\alpha} 2) \cdot(1-\sinh (H t))^{3 / 2}} \mathrm{~d} t \\
& \approx \frac{t_{M}}{2} \cdot\left(\mathrm{e}^{i \cdot(\tilde{\alpha}) \cdot t_{M}-(\tilde{\alpha} 2) \cdot\left(1-\sinh \left(H \cdot t_{M}\right)\right)^{3 / 2}}-1\right) \\
& \Psi_{1, \kappa=n=0} \approx \sqrt{\frac{\omega}{\pi}} \cdot\left[\frac{1}{\omega+i \cdot(t+r)}-\frac{1}{\omega+i \cdot(t-r)}\right] \\
& \tilde{\alpha} 1=\left[\frac{c^{4} \cdot \pi}{16 G \hbar} \cdot\left(r-r^{3} \Lambda_{0}\right)\right], \quad \tilde{\alpha} 2=\frac{\pi}{2 G H^{2}}
\end{aligned}
$$

We will be looking at comparing the real values of Equation (23) in order to obtain a bound on the cosmological constant, and in doing so we have employing the following Equation (11), Equation (20), Equation (21), Equation (22) and Equation (23) in order to get a bound on the Cosmological constant as given by: 


$$
\begin{aligned}
\Lambda_{0} \approx & \tilde{B}^{-2}-\frac{16}{\pi} \cdot \tilde{B}^{-2} \cdot\left(\tilde{\alpha} 2 \cdot(1-\sinh (H \cdot \tilde{B}))^{3 / 2}\right) \\
& -\frac{16}{\pi} \cdot \tilde{B}^{-2} \cdot \cos ^{-1}\left[\frac{2 \cdot 8^{3 / 4} \cdot \pi^{1 / 4}}{8 \pi+(1+\tilde{B})^{2}}-\frac{2 \cdot 8^{3 / 4} \cdot \pi^{1 / 4}}{8 \pi+(1-\tilde{B})^{2}}\right]
\end{aligned}
$$

In doing this, taking into account the Planck units and their normalization, we also need to keep in consideration the frequency, which we will denote here as:

$$
\omega_{\text {signal }} \approx \frac{k_{B} \cdot \sqrt{M_{\text {Planck }} H}}{\hbar \sqrt{1.66 \sqrt{g_{*}}}} \underset{\hbar=\ell_{P}=G=t_{P}=k_{B}=1}{\longrightarrow} \frac{\sqrt{H}}{\sqrt{1.66 \sqrt{g_{*}}}} \approx \frac{T_{\text {temperature }}}{2}
$$

Whereas what we will be doing, after we obtain a frequency of a signal near the mouth of a wormhole is to use the following scaling of frequency, near Earth Orbit from this wormhole. First if the wormhole is right at the start of the Universe [8], we use:

$$
\begin{aligned}
& \left(1+z_{\text {initial era }}\right) \equiv \frac{a_{\text {today }}}{a_{\text {initial era }}} \approx\left(\frac{\omega_{\text {Earth orbit }}}{\omega_{\text {initial era }}}\right)^{-1} \\
& \Rightarrow\left(1+z_{\text {initial era }}\right) \omega_{\text {Earth orbit }} \approx 10^{25} \omega_{\text {Earth orbit }} \approx \omega_{\text {initial era }}
\end{aligned}
$$

If we are say far closer to the Earth, or the Solar system, then we would likely see $[8]$

$$
10 \cdot \omega_{\text {Earth orbit signal }} \approx \omega_{\text {wormhole mouth signal }}
$$

Our derivation so far is to obtain the initial signal frequency for Equation (26) and Equation (27). Our next task is to obtain some considerations as to the Polarization, of say GW to observe and look for, in conclusion of this document.

\section{The Big Picture, Polarization of Signals from a Wormhole Mouth May Affect GW Astronomy Investigations}

We will be referencing [16] and [17], i.e. for [16] we have a rate of production from the wormhole mouth we can quantify as

$$
\Gamma \approx \exp \left(\omega_{\text {signal }} / T_{\text {temperature }}\right)
$$

Whereas we have from [17] a probability for "scalar" particle production from the wormhole given by

$$
\Gamma \approx \exp \left(-E / T_{\text {temperature }}\right)
$$

Whereas if we assume that there is a "negative temperature in Equation (28) and say rewrite Equation (29) as obeying having:

$$
\left(\omega_{\text {signal }} / T_{\text {temperature }}\right) \approx\left(-E / T_{\text {temperature }}\right)
$$

This is specifying a rate of particle production from the wormhole. And so then, whereas what we are discussing in Equation (28) and Equation (29) is having a rate of, from a wormhole mouth, presumably from graviton produc- 
tion. If as an example, we are examining the mouth of a wormhole as being equivalent of a linkage between two black holes, or a black hole-white hole pair, we are presuming a release from the mouth of the wormhole commensurate with looking at [18] with an eye to "white holes" for a black hole model as of probability for "scalar" particle production given as, if $M$ is the mass of the black (white) hole, $m$ is the mass of an emitted "particle", $\omega$ is frequency of emitted particles,

$$
\Gamma \propto \exp \left(-8 \pi M \cdot \omega \cdot\left[1+\frac{\beta}{4} \cdot\left(m^{2}+4 \omega^{2}\right)\right]\right)
$$

Whereas we define the parameter $\beta$ via a modified energy expression, as in [18] given by $\tilde{E}$ as a modified energy expression in [18] [19],

$$
\tilde{E}=E \cdot\left(1-\beta \cdot\left(p^{2}+m^{2}\right)\right)
$$

Our Equations (28) and (29), which are for wormholes, should encompass the same information of Equation (31) which would be consistent with a white hole [20] [21] at the mouth of a wormhole, as would be expected from Equation (31), whereas reviewing a linkage between black holes and white holes as may be for forming a wormhole may give more credence to the information loss criteria as given in [22].

Our next step is to ask if this permits speaking of say GW polarization in the mouth of a wormhole.

To do this, first of all, note that in [23] that the simplest version of a wormhole is one of two universes connected by a "throat" of the form of a "ball" given by $\pi b^{2}$, whereas the term $b$, is in a diagram, consigned to be the radius, or shape of the initial "ball" joining two "universes".

In the case of extending b to become the "shape" of the mouth of a wormhole, we would likely be using [24] for what is called by Visser the "shape" function of the wormhole [25], whereas what we are referring to in Equation (33) comes straight from [23]:

$$
b(r)=\left[r_{0}^{\frac{\gamma-1}{\gamma}}+\gamma \cdot \frac{(8 \pi G)^{\frac{\gamma-1}{\gamma}}}{\tilde{\tilde{\omega}}^{1 / \gamma}} \cdot\left(r^{3}-r_{0}^{3}\right)\right]^{\frac{\gamma}{\gamma-1}} \underset{r \rightarrow r_{0}}{\longrightarrow} r_{0}
$$

Whereas we need to keep in mind the equation of state for pressure and density of [24]

$$
p=\tilde{\tilde{\omega}}(r) \cdot \rho
$$

The long and short of it is as follows. Following [24] we have that:

$$
\rho_{\tilde{\alpha}}=\frac{M}{(4 \pi \tilde{\alpha})^{3 / 2}} \cdot \exp \left(-r^{2} / 4 \tilde{\alpha}\right)
$$

Whereas the $b$ coefficient in the case of Noncommutative geometry is chosen [26]: 


$$
\begin{aligned}
b(r) & =\frac{2 r_{s}}{\sqrt{\pi}} \cdot \hat{\gamma}\left(\frac{3}{2}, \frac{r^{2}}{4 \tilde{\alpha}}\right) \\
& \equiv \frac{2 r_{s}}{\sqrt{\pi}} \cdot\left(\frac{r^{2}}{4 \tilde{\alpha}}\right)^{3 / 2} \cdot \tilde{\Gamma}(3 / 2) \cdot \mathrm{e}^{-3 / 2} \cdot \sum_{k=0}^{\infty}\left(\frac{\left(\frac{r^{2}}{4 \tilde{\alpha}}\right)^{k}}{\tilde{\Gamma}((3 / 2)+k+1)}\right)
\end{aligned}
$$

This is called the incomplete lower gamma function, with $\tilde{\Gamma}$ being a gamma function [27].

From here, using that Equation (36) is to be included in the following metric, as given by the coefficient $[\tilde{\alpha}]=\left[r^{2}\right]$ in terms of dimensional analysis is chosen so that the dimensions of $[\tilde{\alpha}]=\left[r^{2}\right]$ are chosen to contain $M$ as mass in a wormhole. i.e. the denominator of Equation (35) $(4 \pi \tilde{\alpha})^{3 / 2}$ is chosen so that $M$ is within the volume of space so subscribed. And this is for line element [26]. With Equation (35) fully described in [26] and [28],

$$
\mathrm{d} S^{2}=-\exp (-2 \Phi(r)) \mathrm{d} t^{2}+\frac{\mathrm{d} r^{2}}{1-b(r) / r}+r^{2} \cdot\left(\mathrm{d} \theta^{2}+\left(\sin ^{2} \theta\right) \mathrm{d} \varphi^{2}\right)
$$

If we refer to black holes, with extra dimension, $n$, of Planck sized mass, we have a lifetime of the value of about:

$$
\begin{aligned}
& \tau \sim \frac{1}{M_{*}}\left(\frac{M_{\mathrm{BH}}}{M_{*}}\right)^{\frac{n+3}{n+1}} \underset{M_{\mathrm{BH}} \approx M_{\text {Planck }}}{\longrightarrow} 10^{-26} \text { seconds } \\
& M_{*} \approx \text { is the low energy scale, } \\
& \text { which could be as low as a few } \mathrm{TeV},
\end{aligned}
$$

The idea would be that there would be $n$ additional dimensions, as given in Equation (38) which would then lay the door open to investigating [29] and [30] in terms of applications, with [30] of additional polarization states to be investigated, as to signals from the mouth of the wormhole. We will next then go into some predictions into first, the strength of the signals, the frequency range, and several characteristics as to the production rate of Planck sized black holes which conceivably could get evicted by use of Equation (28), in terms of what could be observed via instrumentation.

\section{A First Order Guess as to the Rate of Production of Planck Sized Black Holes through a Wormhole, Using Equation (28)}

In order to do this, we will be estimating that the temperature would be of the order of Planck temperature, i.e. using ideas from [30] and [31],

$$
\frac{\omega_{p}}{T_{p}} \equiv \frac{\sqrt{G k_{B}^{2}}}{\hbar} \underset{\hbar=G=k_{B}=1}{\longrightarrow} 1
$$

If so, then there would be to first order the following rate of production,

$$
\Gamma_{\text {rate of production }} \approx e \approx 2-3
$$


Some of the considerations given in this could be related to [32] as an afterthought whereas the author in [33] estimated for an LHC that there would be about 3000 gravitons produced per second. Assuming a figure from [34] as to the percentage of black hole mass decaying into gravitons, i.e. [34], i.e., 1/1000 of the mass of a Planck sized black hole would delve into gravitons, so if one had 3000 gravitons produced per second, as measured on Earth, one would likely have 2 - 3 black holes, of mass of about $10^{-5}$ grams per black hole, producing say $10^{57}$ gravitons, produced per black hole of mass about $10^{-62}$ grams per black hole [35].

Having said, that what about frequencies? Here, if we have a wormhole throat of about 2 - 3 Planck lengths in diameter, with a frequency of emitted gravitons of about $10^{19} \mathrm{GHz}$ initially, it is realistic, using the following, to expect in many cases a redshift downscaling of frequencies of about $10^{-18}$, if the wormholes are close to the initial near singularity, so then that we could be looking at approximately 10 to $12 \mathrm{GHz}$, on Earth, for frequencies, of initially about $10^{19} \mathrm{GHZ}$. So then note at inflation we have:

$$
\begin{aligned}
& \left(1+z_{\text {initial era }}\right) \equiv \frac{a_{\text {today }}}{a_{\text {initial era }}} \approx\left(\frac{\omega_{\text {Earth orbit }}}{\omega_{\text {initial era }}}\right)^{-1} \\
& \Rightarrow\left(1+z_{\text {initial era }}\right) \omega_{\text {Earth orbit }} \approx 10^{25} \omega_{\text {Earth orbit }} \approx \omega_{\text {initial era }}
\end{aligned}
$$

In our situation, the figure would likely be instead of $10^{25}$ times Earth orbit detected frequency, something closer to $10^{18}$ to $10^{19}$ times Earth orbit GW frequencies detected as given by [36]. The relative GW strength of the signal, if one uses [36] while assuming approximately 10 to $12 \mathrm{GHz}$, for initially about $10^{19}$ $\mathrm{GHz}$ GW signals would be about $h \sim 10^{-26}$ and this could change an order of magnitude given instrument sensitivity. In any case it would be well worth our while to look closely at [37] [38] [39] [40] for additional clues and insights to consider while commencing this investigation. As well as details are given in [41].

\section{Conflicts of Interest}

The author declares no conflicts of interest regarding the publication of this paper.

\section{References}

[1] Weber, J. (2004) General Relativity and Gravitational Waves. Dover Publications, Incorporated, Mineola, New York.

[2] Lu, H.Q., Fang, W., Huang, Z.G. and Ji, P.Y. (2008) The Consistent Result of Cosmological Constant from Quantum Cosmology and Inflation with Born-Infeld Scalar Field. The European Physical Journal C, 55, 329-335. https://doi.org/10.1140/epjc/s10052-008-0564-z

[3] Utpal, S. (2008) Particle and Astroparticle Physics. Taylor \& Francis Group, New York City.

[4] Popov, A.A. and Sushkov, S.V. (2001) Vacuum Polarization of a Scalar Field in 
Wormhole Spacetimes. Physical Review D, 63, Article ID: 044017

https://doi.org/10.1103/PhysRevD.63.044017

https://arxiv.org/abs/gr-qc/0009028

[5] DeBenedictis, A. and Das, A. (2001) On a General Class of Wormhole Geometries. Classical and Quantum Gravity, 18, 1187-1204. arXiv:gr-qc/0009072. https://doi.org/10.1088/0264-9381/18/7/304

[6] Albert, E. and Nathan, R. (1935) The Particle Problem in the General Theory of Relativity. Physical Review, 48, 73-77. https://doi.org/10.1103/PhysRev.48.73

[7] Visser, M. (2002) The Quantum Physics of Chronology Protection. https://arxiv.org/abs/gr-qc/0204022

[8] Lightman, A., Press, W., Price, R. and Teukolsky, S. (1975) Problem Book in Relativity and Gravitation. Princeton University Press, Princeton.

[9] Kolb, E. and Turner, M. (1990) The Early Universe. Frontiers in Physics Lecture Note Series, Addison-Wesley Publishing Company, Menlo Park.

[10] McCracken, D.D. and Dorn, W.S. (1977) Numerical Methods and FORTRAN Programming with Application in Engineering and Science. Publishing House Mir, Moscow, $584 \mathrm{p}$.

[11] Davis, P.J. and Philip, R. (2007) Methods of Numerical Integration. 2nd Edition, Dover Publishers, New York.

[12] Zwillinger, D. (2003) CRC Standard Mathematical Tables and Formulae. 31st Edition, Chapman and Hall/CRC, New York. https://doi.org/10.1201/9781420035346

[13] Kieffer, K. (2012) Quantum Gravity. 3rd Edition, Oxford Science Publications, Oxford University Press, Oxford.

[14] Barrow, J.D. (2002) The Constants of Nature, from Alpha to Omega-The Numbers That Encode the Deepest Secrets of the Universe. Pantheon Books, New York.

[15] Planck, M. (1899) Über irreversible Strahlungsvorgänge. Sitzungsberichte der Königlich Preußischen Akademie der Wissenschaften zu Berlin, 5, 440-480.

[16] Martın-Moruno, P. and Gonzalez-Diaz, P.F. (2011) Thermal Radiation from Lorentzian Traversable Wormholes. Spanish Relativity Meeting (ERE 2010): Gravity as a Crossroad in Physics. Journal of Physics. Conference Series, 314, Article ID: 012037. https://doi.org/10.1088/1742-6596/314/1/012037 https://iopscience.iop.org/article/10.1088/1742-6596/314/1/012037/pdf

[17] Gecim, G. and Sucu, Y. (2020) Quantum Gravity Correction to Hawking Radiation of the 2+1-Dimensional Wormhole. Advances in High Energy Physics, 2020, Article ID: 7516789, 10 p. https://doi.org/10.1155/2020/7516789

https://www.hindawi.com/journals/ahep/2020/7516789/

[18] Chen, D., Wu, H., Yang, H. and Yang, S. (2014) Effects of Quantum Gravity on Black Holes. International Journal of Modern Physics A, 29, Article ID: 1430054. https://doi.org/10.1142/S0217751X14300543 https://arxiv.org/pdf/1410.5071.pdf

[19] Greiner, W. (2000) Relativistic Quantum Mechanics: Wave Equation. Springer-Verlag, Berlin, Heidelberg. https://doi.org/10.1007/978-3-662-04275-5

[20] Retter, A. and Heller, S. (2012) The Revival of White Holes as Small Bangs. New Astronomy, 17, 73-75. arXiv:1105.2776.

https://doi.org/10.1016/j.newast.2011.07.003

[21] Popławski, N.J. (2010) Radial Motion into an Einstein-Rosen Bridge. Physics Letters $B$, 687, 110-113. https://doi.org/10.1016/j.physletb.2010.03.029 
[22] Mathur, S.D. (2008) Resolving the Information Loss Paradox. https://arxiv.org/abs/0810.4525

[23] Gonzalezx, J.A., Guzman, F.S. and Sarbach, O. (2008) On the Instability of Static, Spherically Symmetric Wormholes Supported by a Ghost Scalar Field. AIP Conference Proceedings, 1083, 208-216. https://doi.org/10.1063/1.3058571

[24] Garranattini, R. and Lobo, F.S.N. (2017) Self-Substained Transversable Wormholes. In: Lobo, F.S.N., Ed., Wormholes, Warp Drives and Energy Conditions, Vol. 189, Springer Nature Publishing Company, Cham, 111-135. https://doi.org/10.1007/978-3-319-55182-1_6

[25] Visser, M. (1996) "Lorentzian Wormholes" from Einstein to Hawking. AIP Press, as done by Springer Verlag Press, New York City.

[26] Abramowitz, M. and Stegun, I. (1965) Handbook of Mathematical Functions: With Formulas, Graphs, and Mathematical Tables. Dover Books, New York City.

[27] Wittaker, E.T. and Watson, G.N. (1927) A Course of Modern Analysis. Cambridge University Press, Cambridge.

[28] Nicolini, P., Smailagic, A. and Spallucci, E. (2006) Non Commutative Geometry Inspired Schwarzschild Black Hole. Physics Letters B, 632, 547-551. https://doi.org/10.1016/j.physletb.2005.11.004

[29] Cheung, K. (2002) Black Hole Production and Extra Large Dimensions. Physical Review Letters, 88, Article ID: 221602.

https://doi.org/10.1103/PhysRevLett.88.221602

https://arxiv.org/abs/hep-ph/0110163

[30] Li, F.-Y., Wen, H., Fang, Z.-Y., Li, D. and Zhang, T.-J. (2020) Electromagnetic Counterparts of High-Frequency Gravitational Waves Having Additional Polarization States; Distinguishing and Probing Tensor-Mode, Vector-Mode, and Scalar-Mode Gravitons. The European Physical Journal C, 80, Article No. 879. https://doi.org/10.1140/epjc/s10052-020-08429-2 https://arxiv.org/abs/1712.00766

[31] Carr, B.J. (2004) Primordial Black Holes-Recent Developments. 22nd Texas Symposium on Relativistic Astrophysics, Stanford, 12-17 December 2004, Article No. 0204. https://arxiv.org/abs/astro-ph/0504034

[32] Sokolov, A.V. and Pshirkov, M.S. (2017) Possibility of Hypothetical Stable Micro Black Hole Production at Future $100 \mathrm{TeV}$ Collider. The European Physical Journal C, 77, Article No. 908. https://doi.org/10.1140/epjc/s10052-017-5464-7

[33] Beckwith, A. (2009) Bounds upon Graviton Mass-Using the Difference between Graviton Propagation Speed and HFGW Transit Speed to Observe Post-Newtonian Corrections to Gravitational Potential Fields. 2009 American Physical Society April Meeting, Denver, 2-5 May 2009.

https://citeseerx.ist.psu.edu/viewdoc/download?doi=10.1.1.302.8755\&rep=rep1\&typ $\underline{e=p d f}$

[34] Carr, B., Winstanley, E. and Calmet, X. (2013) Quantum Black Holes. Springer Briefs in Physics, Springer-Nature, Heidelberg, Germany.

[35] Will, C.M. (1998) Bounding the Mass of the Graviton Using Gravitational-Wave Observations of Inspiralling Compact Binaries. Physical Review D, 57, 2061-2068. arXiv:gr-qc/9709011. https://doi.org/10.1103/PhysRevD.57.2061

[36] Maggiore, M. (2008) Gravitational Waves, Vol. 1: Theory and Experiment. Oxford University Press, Oxford.

[37] Hawking, S. and Ellis, G.F.R. (1973) The Large Scale Structure of Space-Time. Cam- 
bridge University Press, Cambridge. https://doi.org/10.1017/CBO9780511524646

[38] Nojiri, S., Odintsov, S.D., Oikonomou, V.K. and Paul, T. (2019) Nonsingular Bounce Cosmology from Lagrange Multiplier $F(R)$ Gravity. Physical Review D, 100, Article ID: 084056. https://doi.org/10.1103/PhysRevD.100.084056 https://arxiv.org/abs/1910.03546

[39] Wang, Q., Zhu, Z. and Unruh, W.G. (2017) How the Huge Energy of Quantum Vacuum Gravitates to Drive the Slow Accelerating Expansion of the Universe. Physical Review D, 95, Article ID: 103504. arXiv:1703.00543. https://doi.org/10.1103/PhysRevD.95.103504

[40] Astier, P., Guy, J., Regnault, N., Pain, R., Aubourg, E., Balam, D., et al. (2006) The Supernova Legacy Survey: Measurement of $\Omega_{\mathrm{M}}, \Omega_{\Lambda}$ and W from the First Year Data Set. Astronomy and Astrophysics, 447, 31-48. arXiv:astro-ph/0510447.

[41] Chen, P. (1994) Resonant Photon-Graviton Conversion: From Earth to Heaven. SLAC PUB 6666 September 1994 (T/E/A).

https://www.slac.stanford.edu/pubs/slacpubs/6500/slac-pub-6666.pdf 